\title{
Sexualerziehung in Jugendwohngruppen
}

\author{
Beteiligungsorientierte Unterstützung der sexuellen Entwicklung von Jugendlichen \\ in der Heimerziehung
}

Beteiligung gilt heute als ein grundlegendes Ideal in der Heimerziehung und auch die erzieherische Unterstützung der sexuellen Entwicklung von Jugendlichen findet breite Anerkennung. Trotzdem wurden diese Bereiche bislang nur sehr begrenzt zusammengebracht. Hier klafft eine Lücke. Wie kann Sexualerziehung in Jugendwohngruppen beteiligungsorientiert gestaltet werden?

K inder und Jugendliche haben ein Recht auf Beteiligung (Kinderrechtskonvention, Art. 12 u. 13), deren Umsetzung daher als Ideal in der Heimerziehung gilt (Krause 2019). Ziel ist es, Kindern und Jugendlichen zu ermöglichen, umfassend über ihre Lebenszusammenhänge mitzuentscheiden (Wolff und Hartig 2013, S. 17 ff.). Ebenso haben sie ein Recht auf die erzieherische Unterstützung ihrer sexuellen Entwicklung durch Sexualerziehung (Mantey 2020). In der stationären Erziehungshilfe kann sie u. a. in Form von individuellen Gesprächen, sexualpädagogischen Bildungsangeboten oder durch die Bereitstellung von Medien und Materialien umgesetzt werden (ebd.). Auch dies findet

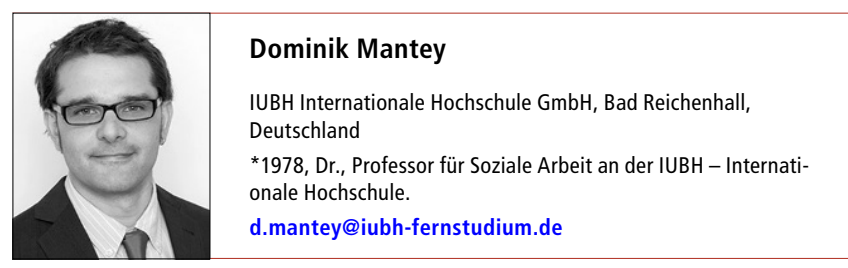

Zusammenfassung Der Beitrag verbindet zwei Herausforderungen der stationären Erziehungshilfe: Beteiligung und Sexualerziehung. Er zeigt, warum auch Sexualerziehung konsequent beteiligungsorientiert gestaltet werden sollte. Außerdem werden Umsetzungsmöglichkeiten für einzelne Bereiche der Sexualerziehung skizziert, etwa für die sexualitätsbezogene Begleitung einzelner Jugendliche sowie für die Gestaltung von sexualpädagogischen Gruppenangeboten. Zugleich werden Grenzen der Beteiligung aufgezeigt, z. B. dort, wo der Schutz der Jugendlichen im Vordergrund stehen muss. Des Weiteren werden besondere Herausforderungen benannt, die sich aus dem Thema „Sexualität“ für die Gestaltung von Beteiligungsprozessen ergeben.

Schlüsselwörter Sexualpädagogik, Heimerziehung, Sexualerziehung, Jugendliche, Beteiligung als Aufgabe stationärer Erziehungshilfe breite Anerkennung (u. a. Bärenz 2014; Hartwig 2015; Müller 2017). Dennoch wurden Beteiligung und Sexualerziehung bislang wenig zusammengedacht. Die Literatur zur Beteiligung in der Heimerziehung enthält zwar auch Aspekte der individuellen Sexualität von Kindern und Jugendlichen, etwa die Mitbestimmung in Bezug auf den eigenen Körper oder die Wahl der Verhütungsmittel (Wolff und Hartig 2013, S. 48), aber ein Großteil der Sexualerziehung als Erziehungspraxis taucht hier nicht auf. Im Folgenden wird aufgezeigt, warum Sexualerziehung in Wohngruppen für Jugendliche grundsätzlich beteiligungsorientiert gestaltet werden sollte und wie eine beteiligungsorientierte Sexualerziehung aussehen kann. Zunächst sieben Gründe für eine beteiligungsorientierte Gestaltung der Sexualerziehung:

1. Jugendliche haben in Wohngruppen ein Recht auf Beteiligung, entsprechend den Artikeln 12 und 13 der Kinderrechtskonvention sowie dem 8. Sozialgesetzbuch. Sie sind in allen sie berührenden Angelegenheiten - also auch in Fragen der Sexualität - anzuhören. Ihre Meinung ist angemessen und ihrem Alter entsprechend zu berücksichtigen (SBG VIII $\$ 8$ Abs. 1).

2. Jugendliche haben ein Recht auf sexuelle Selbstbestimmung, entsprechend Artikel 5 der „Sexual and Reproductive Health Rights“ (IPPF 2008). Im Kontext der Heimerziehung bedeutet die Realisierung dieses Rechts, Jugendliche selbst über alle Aspekte ihrer Sexualität, also auch über Sexualerziehung, entscheiden zu lassen, solange dem keine anderen Interessen, wie z. B. Schutz der Jugendlichen, entgegenstehen.

3. Lern- und Bildungsprozesse werden durch Beteiligung realisiert (Wolff und Hartig 2013, S. 37): Zum einen setzen sich Jugendliche im Rahmen von 
Beteiligungsprozessen mit den Inhalten der Beteiligung auseinander, eignen sich z. B. den Aspekt der Intimität an, wenn sie in einer Gruppensitzung über Regeln des Zugangs zu Zimmern diskutieren. Zum anderen fördert Beteiligung ihre soziale Kompetenz, weil ein anerkennender Umgang miteinander gelernt wird (ebd., S. 37).

4. Beteiligung hat eine gewaltpräventive Wirkung, da sie strukturell für eine Verringerung des institutionellen Machtgefälles zwischen Erziehenden und Jugendlichen sorgt (Rosenbauer und Stremmer 2017, S. 154). Meinungen und Kritik werden gefördert und systematisch gehört (Wolff und Hartig 2013, S. 37). So kann in Beteiligungsprozessen ein Verhalten von Erziehenden thematisiert werden, das Jugendliche als sexuell grenzverletzend wahrnehmen.

5. Sexualerziehung gewinnt durch Beteiligung überhaupt erst an Qualität, da sie individuell angepasst werden kann. Dies bezieht sich einerseits auf die thematisierten Inhalte: Dass Medien und Materialien in der Wohngruppe zu ihren Entwicklungsherausforderungen und Bedürfnissen passen, wird durch Beteiligung der Jugendlichen sichergestellt (Mantey 2019). Andererseits betrifft dies die individuellen Grenzen der Jugendlichen: Indem sie z. B. über ihre Ansprechpartner_innen oder die Themen von Entwicklungsgesprächen zu Sexualität mitentscheiden, werden Grenzen der Intimität berücksichtigt und schamauslösende Situationen vermieden (ebd.).

6. Beteiligung hat eine Wirkung auf die Beziehung zwischen Jugendlichen und Erziehenden, denn sie schafft Vertrauen. Dies ist eine wichtige Grundlage, um Sexualerziehung überhaupt erst zu ermöglichen (Mantey 2017, S. 215 ff.).

7. Sexualerziehung kann im professionellen Sinne ohne Beteiligung in vielen Fällen gar nicht stattfinden, da Jugendliche ihre Sexualität verbergen, wenn sie in ihren Entscheidungen eingegrenzt werden (Mantey 2015).

Die einzelnen Elemente der Sexualerziehung und die jeweiligen Möglichkeiten und Grenzen der Umsetzung von Beteiligung werden nachfolgend exemplarisch dargestellt.

\section{Individuelle Begleitung}

Ein zentraler Bereich der Sexualerziehung umfasst die individuelle Begleitung einzelner Jugendlicher hinsichtlich ihrer sexuellen Entwicklung (Mantey 2020). Realisiert wird dies v. a. über individuelle Gespräche, meist durch Bezugserziehende. Dabei werden u. a. Herausforderungen und Kompetenzen der/des Jugendlichen besprochen, individuelle Regeln entwickelt oder Ressourcen für die sexuelle Entwicklung geklärt.

Ein Beispiel: Der 14-jährige Julian wird im Aufnahmeprozess über Sexualerziehung in der Wohngruppe und Beteiligungsverfahren hierbei informiert. Wenn er nicht von sich aus auf seinen Bezugsbetreuer zugeht, wird er - wie angekündigt - nach wenigen Monaten gefragt, ob er bereits genügend Vertrauen gefasst hat und mit wem er sich vorstellen könnte, über Aspekte seiner Sexualität zu sprechen. Da er zu seinem Bezugsbetreuer bereits genügend Vertrauen hat, klärt er mit diesem, wo und wann das Gespräch stattfinden soll. Im Gespräch orientiert sich der Erziehende an einem Leitfaden (ebd.), der verschiedene Themen vorschlägt. Die Entscheidungen, welche Themen besprochen werden und welche ihm zu intim sind, trifft jedoch der Jugendliche. Er entscheidet u. a. mit, welche Informationen etwa an seine Eltern oder andere Erziehende weitergegeben werden.

Die Umsetzung von Partizipation im Rahmen individueller Begleitung bedeutet daher u. a., dass Jugendliche mitbestimmen

- über ihre_n Ansprechpartner_in zu Aspekten der Sexualität,

- über Zeitpunkt und Rahmen der Gespräche,

- über die Inhalte von Gesprächen,

- darüber, welche ihre Sexualität betreffenden Informationen an das Team, ihre Eltern oder das Jugendamt weitergegeben werden.

Wie weit die Entscheidungsbefugnis der Jugendlichen reicht, also wie viel Macht sie von den Erziehenden eingeräumt bekommen, ist jedoch vom Einzelfall abhängig. Sie muss u. U. eingeschränkt werden, wenn etwa der Schutz der betreffenden oder der anderen Jugendlichen in der Wohngruppe realisiert werden muss. Zeigt ein Jugendlicher z. B. sexuell grenzverletzendes Verhalten, kann bezüglich des Zeitpunkts nicht gewartet werden, bis er aus seiner Sicht ausreichend Vertrauen gefasst hat - Gespräche müssen sofort stattfinden.

Ebenso müssen Informationen, die für den Schutz der Jugendlichen wichtig sind, an das Team und auch an die Eltern weitergeleitet werden - unabhängig davon, ob die/der Jugendliche das möchte oder nicht. Die Beteiligungsmöglichkeiten müssen also u. U. eingeschränkt werden. Dafür bedarf es eines transparenten Verfahrens, das z. B. in einer sexualpädagogischen Konzeption festgehalten werden kann. 


\section{Gruppenangebote}

In sexualpädagogischen Bildungsveranstaltungen, die sich an die gesamte Gruppe der Jugendlichen wenden (Mantey 2020), können mittels sexualpädagogischer Methoden z. B. sexualisierte Gewalt oder die Pubertät thematisiert werden. Auch in diesem Bereich der Sexualerziehung kann Partizipation auf vielfältige Weise umgesetzt werden.

Ein Beispiel: Die Erzieherin Johanna plant eine sexualpädagogische Gruppenveranstaltung zur Pubertät. Zuvor informiert sie die Jugendlichen über die Veranstaltung und stellt eine Box auf. Hier können die Jugendlichen Fragen hinterlegen, mit deren Hilfe die Erzieherin die Themen der Veranstaltung anpasst. Auch während der Durchführung bleibt Johanna mit den Jugendlichen im Austausch und berücksichtigt ihre Wünsche. Anfangs einigen sie sich auf Regeln für die Veranstaltung, danach entscheiden die Jugendlichen mit über den Verlauf. Je nach ihren Interessen werden bestimmte Themen vertieft und unterschiedliche mediale Möglichkeiten angeboten, z. B. kann zwischen Quiz und Film entschieden werden.

Die Umsetzung der Partizipation im Rahmen sexualpädagogischer Gruppenangebote bedeutet also z. B., dass Jugendliche mitbestimmen über

- die Themen der Veranstaltungen,

- den Verlauf der Veranstaltungen (etwa, welche Inhalte nur kurz oder welche ausführlich thematisiert werden),

- die innerhalb der Veranstaltungen genutzten Methoden,

- die Regeln für die Zusammenarbeit in der Veranstaltung.

Auch in diesem Bereich stellt der Schutz der Jugendlichen eine wichtige Grenze dar. Bei Veranstaltungen bzw. Themen, die für den Schutz der Jugendlichen sehr wichtig sind, dürfen sie nur begrenzt mitentscheiden, da nur durch verbindliche Durchführung und Teilnahme eine Vermittlung schutzrelevanter Informationen an die Jugendlichen sichergestellt werden kann. So sollten in jeder Wohngruppe Veranstaltungen zu den Themen Verhütung, sexuelle Vielfalt und sexualisierte Gewalt durchgeführt werden, da sie wichtige Schutzfunktionen erfüllen (Mantey 2020). Trotz der Verbindlichkeit dieser Veranstaltungen muss dabei auf Mitbestimmung nicht verzichtet werden. Wichtige Voraussetzung ist die Klärung, welche Inhalte zwingend zu vermitteln sind und welche je nach Interessen der Jugendlichen thematisiert werden, bei nicht verpflichtenden Inhalten können sie mitentscheiden.
Regeln

$\mathrm{Zu}$ Themen, die meist reguliert werden, gehören u. a. sexuelle Grenzverletzungen und Gewalt, Liebesbeziehungen in der Gruppe, Privatsphäre, Umgang mit intimen Informationen, Sprache, der Empfang von Besuch über Nacht, Ausgabe von Verhütungsmitteln oder die Ausgabe oder Nutzung sexualpädagogischer Medien und Materialien (Rusack 2015; Domann et al. 2015; Mantey 2017). Es lassen sich zwei Arten von Regeln unterscheiden: „Einerseits sind allgemeingültige Regeln notwendig, die beispielsweise klare Grenzen ausweisen, wo Gewalt anfängt und wie damit umzugehen ist. Andererseits werden innerhalb eines Spielraums, den die allgemeingültigen Regeln lassen, individuelle Entscheidungen benötigt, die an den/die jeweilige Jugendliche_n angepasst sind“ (Mantey 2020, i.E.). Für beide Regelebenen sind spezifische Beteiligungsprozesse festzulegen. Während einige individuelle Regeln im Austausch zwischen den jeweiligen Erziehenden und Jugendlichen festgelegt werden können, etwa bezüglich der Nutzung bestimmter Bücher oder Internetseiten zur Sexualaufklärung, benötigen andere Regeln die Mitentscheidung der gesamten Gruppe, aller Erziehenden und der Leitung oder auch der Eltern - etwa, wenn es um Übernachtungsbesuche in der Gruppe geht.

Die Umsetzung der Partizipation im Rahmen sexualitätsbezogener Regeln bedeutet also z. B., dass Jugendliche mitbestimmen über

- ausschließlich sie betreffende Regeln, etwa zum Besuch von Freund_innen auf dem eigenen Zimmer,

- die gesamte Gruppe betreffende Regeln, etwa zum Umgang mit Intimität oder die Verwendung von sexueller Sprache.

Auch bezüglich der Beteiligung in der Entwicklung von Regeln bestehen Grenzen. So müssen die Erziehenden den Schutz von in der Gruppe wohnenden Kindern gewährleisten, wenn z. B. die Jugendlichen frei zugängliche Medien der Sexualaufklärung wünschen. Weitere Grenzen können in Rechtsnormen liegen, die es den Erziehenden unmöglich machen, bis zu einem bestimmten Alter Übernachtungsbesuche zu erlauben (Mantey 2020). Auch generelle Orientierungen der Einrichtung können Grenzen der Beteiligung darstellen, wenn es z. B. um Sprachregelungen geht und die Jugendlichen den Begriff „schwul“ als Schimpfwort nutzen möchten, die Einrichtung sich jedoch gegen Diskriminierung und Ausgrenzung von LGBTQ-Personen richtet.

\section{Medien}

Auch in Bezug auf die Nutzung von Medien der Sexualaufklärung bzw. sexuellen Bildung sollten Jugend- 
lichen Beteiligungsmöglichkeiten eingeräumt werden. So kann z. B. über die Anschaffung von Büchern oder die Freigabe von Internetseiten mittels einer Whitelist auf dem Gruppen-PC diskutiert werden. Voraussetzung sind bereits bestehende Medienkompetenzen der Jugendlichen bzw. Zeit und Raum für die Vermittlung von Medienkompetenzen in Beteiligungsprozessen. Gemeinsame Entscheidungen über die Medien in der Gruppe können auch das Ergebnis eines sexualpädagogischen Gruppenangebots zu sexualitätsbezogenen Medien mit dem Ziel der Medienkompetenzschulung sein. Auch über Zugänge zu Büchern und anderen Medien, etwa vor dem Hintergrund des Schutzes der Intimität, können Jugendliche mitentscheiden.

Grenzen der Beteiligung liegen z. B. darin, dass Internetseiten oder Broschüren und Bücher, die sich an LGBTQ-Jugendliche richten, auch dann zur Verfügung gestellt werden müssen, wenn die Jugendlichen dies ablehnen. Hierbei geht es um Schutz und Unterstützung dieser Jugendlichen, auch wenn sie sich nicht als solche „geoutet" haben. Der Zugang zu einzelnen Medien muss so geregelt werden, dass Kinder oder jüngere Jugendliche vor Medieninhalten geschützt werden, die ihrem Alter nicht entsprechen.

\section{Gestaltung partizipativer Prozesse}

Für alle Bereiche der Sexualerziehung sind je nach institutionellen Gegebenheiten - wie z. B. bereits gegebenen Partizipationsverfahren, Hierarchiestrukturen - Verfahren zu entwickeln, um die jeweilige Machtverteilung und Entscheidungsbefugnis festzulegen. Dies ist wichtig, damit in kritischen Situationen - wenn es in der Wohngruppe z. B. zu einer sexuellen Grenzverletzung kommt - nachvollzogen und dargelegt werden kann, wie und unter Einbezug welcher Informationen und Akteur_innen welche Entscheidung getroffen wurde. Orte, Situationen, Zeiträume, Gremien und Personen der Entscheidungsfindung sollten in einer sexualpädagogischen Konzeption festgelegt sein.

Grundsätzlich sollte die Expertise der Fachkräfte darüber entscheiden, welche Entscheidungsgewalt in welcher Situation abgegeben werden kann und wo die jeweiligen Grenzen liegen. Abhängig ist dies u. a. vom Alter der Jugendlichen, von sexualitätsbezogenen Vorerfahrungen und Gefährdungslagen oder von der Gruppenzusammensetzung. Dies bedeutet auch, sexualitätsbezogene Entscheidungen in regelmäßigen Abständen erneut vorzulegen. Regelungen wie der Zugang zu Medien oder zu Übernachtungsbesuchen müssen wiederholt neu getroffen werden.

Im Vergleich zu anderen Themen der Partizipation ist bezüglich der Gestaltung der Beteiligungsprozesse zu beachten, dass es sich hierbei auch um sexualpädagogische Situationen handelt, da Sexualität thematisiert wird, und dies bestimmte Konsequenzen hat, da Sexualität eben „kein Thema wie jedes andere“ ist. Sexualpädagogische Situationen erfordern u. a. den Schutz von Intimität sowie einen reflektierten und sensiblen Umgang mit Scham, da Sexualität in großem Maße tabuisiert ist. Daher sind auch Regeln zu formulieren, welche die Kommunikation innerhalb des Beteiligungsprozesses regeln und sicherstellen, dass der Prozess selbst nicht zu Grenzverletzungen führt. So dürfen durch Jugendliche oder Erziehende unautorisiert keine intimen Informationen über andere Jugendliche preisgegeben werden. Es bedarf also ähnlicher Regeln wie für die Gestaltung sexualpädagogischer Gruppenveranstaltungen (Mantey 2020).

\section{Fazit}

Im Kontrast zu den aktuellen Diskursen um Partizipation, Heimerziehung und Sexualpädagogik, in denen eine Umsetzung von Beteiligung in Bezug auf Sexualerziehung nur sehr bedingt thematisiert wird, zeigt dieser Beitrag die vielfältigen Gründe auf, die für eine systematische Umsetzung von Partizipation bei der Sexualerziehung sprechen. Anhand von Beispielen wurde die Vielfalt der Umsetzungsmöglichkeiten skizziert. Gleichwohl wurden - wie für alle institutionellen Kontexte der Kinder- und Jugendhilfe - Grenzen der Partizipation aufgezeigt, etwa dort, wo der Schutz von Jugendlichen realisiert werden muss.

Zudem wurde die Notwendigkeit deutlich, Prozesse der Partizipation zu klären, z. B. in einem sexualpädagogischen oder beteiligungsorientierten Konzept. Hierbei ist zu berücksichtigen, dass Beteiligungsprozesse zu Aspekten der Sexualität keine „gewöhnlichen“ Beteiligungsprozesse sind, sondern sexualpädagogische Situationen, die besondere Herausforderungen mit sich bringen.

Eingegangen. 11. Februar 2020

Angenommen. 25. Februar 2020

Funding. Open Access funding provided by Projekt DEAL.

Open Access. Dieser Artikel wird unter der Creative Commons Namensnennung 4.0 International Lizenz veröffentlicht, welche die Nutzung, Vervielfältigung, Bearbeitung, Verbreitung und Wiedergabe in jeglichem Medium und Format erlaubt, sofern Sie den/die ursprünglichen Autor(en) und die Quelle ordnungsgemäß nennen, einen Link zur Creative Commons Lizenz beifügen und angeben, ob Änderungen vorgenommen wurden.

Die in diesem Artikel enthaltenen Bilder und sonstiges Drittmaterial unterliegen ebenfalls der genannten Creative Commons Lizenz, so- 
fern sich aus der Abbildungslegende nichts anderes ergibt. Sofern das betreffende Material nicht unter der genannten Creative Commons Lizenz steht und die betreffende Handlung nicht nach gesetzlichen Vorschriften erlaubt ist, ist für die oben aufgeführten Weiterverwendungen des Materials die Einwilligung des jeweiligen Rechteinhabers einzuholen.

Weitere Details zur Lizenz entnehmen Sie bitte der Lizenzinformation auf http://creativecommons.org/licenses/by/4.0/deed.de

\section{Literatur}

Bärenz, P. (2014). Es geht um das „und“. Erarbeitung eines sexualpädagogischen Konzeptes im Pestalozzi Kinder- und Jugenddorf e. V. ajs - informationen; Fachzeitschrift der Aktion Jugendschutz, 2014(1), 26-30.

Domann, S., Eßer, F., Rusack, T., Klepp, N., \& Löwe, C. (2015). Jugendliche in der Heimerziehung zwischen Verboten, informellen Regeln und Klatsch: Umgangsweisen mit Körperkontakt. neue Praxis, 45(5), 503-518.

Hartwig, L. (2015). Mädchen-Sein und Sexualpädagogik in der stationären Erziehungshilfe. Forum Erziehungshilfen, 21(2), 75-79.

International Planned Parenthood Federation (IPPF) (2008). Sexuelle Rechte: Eine IPPF-Erklärung. https://www.ippf.org/sites/default/files/ippf_ sexual_rights_declaration_german.pdf.Zugegriffen: 19. Dez. 2019.

Krause, H.-U. (2019). Beteiligung als umfassende Kultur in den Organisationen der Hilfen zur Erziehung. Haltungen-Methoden-Strukturen. Frankfurt/Main: IGfH.

Mantey, D. (2015). Sexualpädagogik in der Heimerziehung? „Ja gerne, aber ich entscheide selbst! “. Einblicke in die Sicht von Jugendlichen. sozialmagazin, 40(1-2), 70-79.

Mantey, D. (2017). Sexualerziehung in Wohngruppen der stationären Erziehungshilfe aus Sicht der Jugendlichen. Weinheim: Juventa.

Mantey, D. (2019). Manchmal ist weniger mehr ... Tabus, Intimität und Scham in der sexualpädagogischen Praxis der Kinder- und Jugendhilfe. Sozial Extra, 39(6), 20-24.

Mantey, D. (2020). Sexualpädagogik und Sexuelle Bildung in der Heimerziehung - Jugendliche individuell und beteiligungsorientiert begleiten . Weinheim: Juventa.

Müller, M. (2017). Heimerziehung und Sexualität. In A. Klein \& E. Tuider (Hrsg.), Sexualität und Soziale Arbeit (Bd. 40, S. 207-229). Baltmannsweiler: Schneider Verlag Hohengehren $\mathrm{GmbH}$.

Rosenbauer, N., \& Stremmer, T. (2017). Macht und Beschämung - verdrängte und verdeckte Dimensionen in Beziehungen zwischen Fachkräften und Adressat innen erzieherischer Hilfen. Forum Erziehungshilfen, 23(3), 151-155.

Rusack, T. (2015). Küssen verboten? Sexualität und Paarbeziehungen aus der Sicht von Jugendlichen in stationären Einrichtungen. Sozial Extra. https://doi.org/10.1007/s12054-015-0080-y

Wolff, M., \& Hartig, S. (2013). Gelingende Beteiligung in der Heimerziehung. Gute Praxis beim Mitreden, Mitwirken und Mitbestimmen von Kindern und Jugendlichen im Heimalltag; ein Werkbuch für Jugendliche und ibre BetreuerInnen. Weinheim: Beltz Juventa.

Hier steht eine Anzeige. Springer 